\title{
Uretrolitíase obstrutiva em equino
}

\author{
Obstructive Urethrolithiasis in a Horse \\ Juliana Targino Silva Almeida e Macêdo', Carmo Emanuel Almeida Biscarde², \\ Karla Alvarenga Nascimento', Jair Alves Ferreira Júnior' \& Pedro Miguel Ocampos Pedroso'
}

\begin{abstract}
Background: The presence of macroscopic concretions in the urinary system is called urolithiasis. In horses the occurrence is rare. The calculi are generally large and unique spicule concretions, composed of calcium carbonate crystals. Less commonly, the concretions are of mixed crystals of calcium carbonate and of calcium phosphate. The aim of the this work was to report a case of obstructive urethrolithiasis in a horse.

Case: A 1-year-and-5-month-old, undefined breed, uncastrated male presented tenesmus, strangury, and dysuria. The horse died seven days after the onset of clinical signs and was referred for necropsy. At necropsy, samples were collected from organs of the abdominal cavity, thoracic cavity, central nervous system and integumentary system. The fragments were fixed in 10\% formaldehyde, cleaved, routinely processed for histology and stained with hematoxylin and eosin. A urinary calculus collected during necropsy was sent for chemical analysis. In the abdominal cavity, a large quantity of citrus liquid of uremic odor was observed. Deposition of fibrin filaments in the peritoneum was observed, as well as in diaphragm and abdominal organs. Serosa of the small and large intestine was red with evidence of the capillaries. Kidneys presented multiple slightly protrusive white areas with a maximum diameter of $3 \mathrm{~mm}$. When cutting, these areas were limited to the cortical region. There was rupture of the urinary bladder, diffuse loss of the mucosa with capillary exposure and evidence, as well as the deposition of fibrin and crude blood clots. The opening of the penile urethra presented a urolith of approximately $3.5 \times 3 \mathrm{~cm}$ in size, with a rough and porous surface, light brown and of a stony consistency. Necrosis of the mucosa and hemorrhage were also observed in the urethra. Microscopically, the kidney showed multiple foci formed by intense inflammatory infiltrate composed of macrophages, neutrophils and plasma cells in the interstitium of the cortical region. In the urinary bladder, there was diffuse necrosis of the lining epithelium, marked inflammatory infiltrate composed mainly of neutrophils in the submucosa, in addition to fibrin deposition and edema. Penile urethra presented changes similar to those described in the urinary bladder. The chemical analysis of the urinary calculus demonstrated the presence of calcium oxalate, triple magnesium ammonia (struvite) phosphate and ammonium in its composition.

Discussion: The diagnosis of obstructive urethrolithiasis was established from the clinical-pathological condition. The occurrence of urolithiasis in horses is rare. The lower occurrence in this species is probably attributed to the large amount of mucus produced by the glands in the renal pelvis and the proximal ureter, which acts as a lubricant to prevent the adhesion of the crystals to the urothelium. The absence of sigmoid flexure and vermiform urethral process in equine also influence the low occurrence of the disease, when compared to ruminants. Rupture of the urinary bladder followed by uroperitonitis and nephritis as observed in this case are common consequences in obstructive urolithiasis. Uroliths in equines are generally composed of a variety of hydrated calcium salts and calcium carbonate, magnesium, and phosphorus salts, with less common struvite compounds. Urethrolithiasis is poorly described in horses and can be caused by struvite stones.
\end{abstract}

Keywords: obstructive urethrolithiasis, equine, urinary system, struvite.

Descritores: uretrolitíase obstrutiva, equino, sistema urinário, estruvita. 


\section{INTRODUÇÃO}

A presença de concreção macroscópica no sistema urinário é denominada urolitíase. Nos equinos a ocorrência é rara [3,6]. Em um estudo retrospectivo em equinos, a urolitíase compreendeu $0,11 \%$ de todos os diagnósticos e 7,8\% das alterações no sistema urinário. Dos 325 equinos com urolitíase, 59,7\% apresentaram cálculo cístico, $24 \%$ cálculo uretral, 12,6\% cálculo renal e 3,7\% cálculo ureteral. Em 10\% dos casos os equinos tinham cálculos em múltiplos locais [5]. Os cálculos geralmente são concreções espiculadas grandes e únicas, compostas de cristais de carbonato de cálcio. Menos comumente, as concreções são de cristais mistos de carbonato de cálcio e de fosfato de cálcio [2]. O objetivo do presente estudo é relatar um caso de uretrolitíase obstrutiva em equino.

\section{CASO}

Um equino de um ano e cinco meses de idade, sem raça definida, macho não castrado, apresentou tenesmo, estrangúria e disúria. Segundo o médico veterinário e o proprietário do equino, não havia história prévia de doença e/ou tratamento. O equino morreu sete dias após o início dos sinais clínicos e foi encaminhado para necropsia. Na necropsia foram coletas amostras de órgãos da cavidade abdominal, cavidade torácica, sistema nervoso central e sistema tegumentar. Os fragmentos foram fixados em formol $10 \%$, clivados, processados de forma rotineira para histologia e corados pela hematoxilina e eosina. Um cálculo urinário coletado durante a necropsia foi enviado para análise de composição química.

Macroscopicamente as mucosas oral e ocular estavam pálidas. Ao abrir a cavidade abdominal

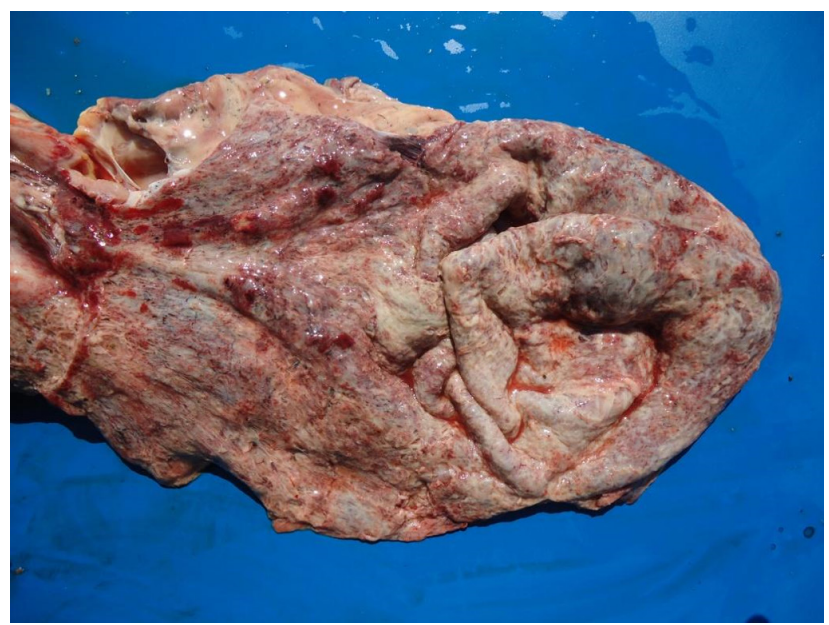

Figura 1. Uretrolitíase em equino. Bexiga urinária. Necrose difusa da mucosa com deposição de fibrina e coágulos de sangue. observou-se grande quantidade de líquido citrino de odor urêmico. Havia deposição de filetes de fibrina no peritônio, diafragma e órgãos abdominais. A serosa do intestino delgado e grosso estava vermelha e com evidenciação dos capilares. Nos rins, apresentavam múltiplas áreas brancas levemente protrusas com diâmetro máximo de $3 \mathrm{~mm}$. Ao corte estas áreas limitavam-se a região cortical. Havia ruptura da bexiga urinária, perda difusa da mucosa com exposição e evidenciação de capilares além da deposição de fibrina e coágulos cruóricos (Figura 1). Na abertura da uretra peniana havia um urólito de aproximadamente 3,5 × $3 \mathrm{~cm}$ de tamanho, de superfície rugosa e porosa, castanho claro e de consistência pétrea (Figura 2). Necrose da mucosa e hemorragia também foi observada na uretra.

Microscopicamente no rim havia múltiplos focos formados por intenso infiltrado inflamatório composto por macrófagos, neutrófilos e plasmócitos no interstício da região cortical. Nestas áreas havia necrose do epitélio de túbulos por vezes com lise da membrana basal associado ao infiltrado inflamatório, além de focos de mineralização, nas camadas cortical e medular. $\mathrm{Na}$ bexiga urinária havia necrose difusa do epitélio de revestimento, acentuado infiltrado inflamatório composto principalmente por neutrófilos na submucosa, além da deposição de fibrina e edema. Nas camadas musculares, interna e externa havia dissociação de fibras pela presença de edema, fibrina e neutrófilos. Na uretra peniana haviam alterações semelhantes as descrita na bexiga urinária.

A análise química do cálculo urinário demonstrou a presença de oxalato de cálcio, fosfato triplo amoníaco magnesiano (estruvita) e amônio em sua composição.

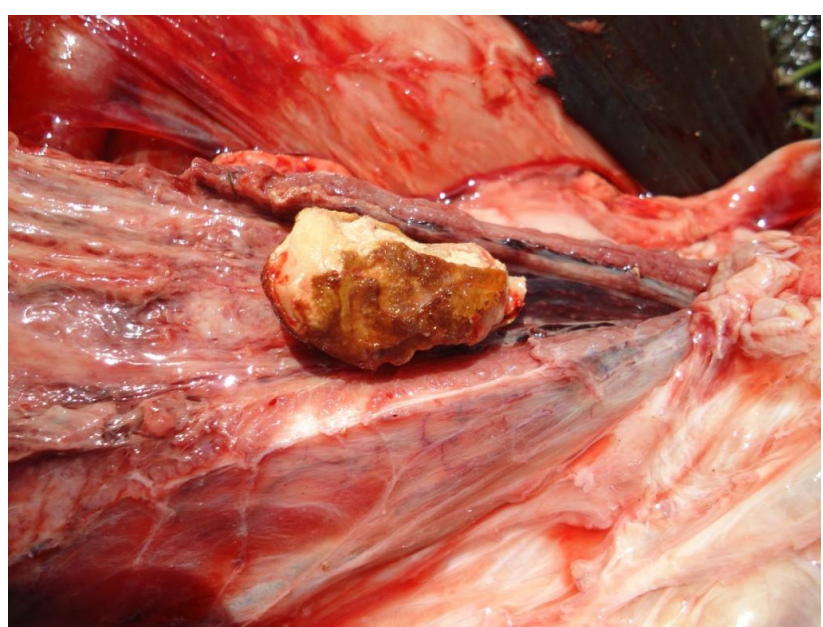

Figura 2. Uretrolitíase em equino. Uretra peniana. Presença de urólito e necrose do epitélio uretral. 


\section{DISCUSSÃO}

O diagnóstico de uretrolitíase obstrutiva foi estabelecido a partir do quadro clínico-patológico. A ocorrência de urolitíase em equinos é rara [3,6]. A abaixa ocorrência nesta espécie provavelmente pode ser atribuída à grande quantidade de muco produzido pelas glândulas na pelve renal e no ureter proximal, desta forma atua como lubrificante para impedir a aderência dos cristais ao urotélio [2]. A ausência da flexura sigmóide e processo uretral vermiforme no equino também influenciam na baixa ocorrência da doença quando comparados aos ruminantes [7]. A manifestação clínica ocorre com maior frequência em machos castrados e com idade entre 2 a 18 anos $[1,4,6]$. No caso descrito, diferencia-se da literatura por ocorrer em um equino não castrado e jovem (1,5 ano). Não foram relatados pelo médico veterinário e pelo proprietário fatores que pudessem contribuir com o surgimento e desenvolvimento do urólito neste equino. No entanto, sabe-se que o desenvolvimento de urólitos em equinos não é bem compreendido, mas células epiteliais descamadas, leucócitos, suturas não absorvíveis e tecidos necróticos foram relatados como núcleos de cálculos císticos. Células resultantes de necrose renal podem forrnecer os núcleos para a formação de urólitos, bem como, necrose papilar renal, secundária à desidratação e terapia com anti-inflamatório não esteróide, pielonefrite, septicemia neonatal, neoplasia e displasias renais congênitas, como doença renal policística [6].
Os sinais clínicos apresentados foram semelhantes aos descritos na literatura [3]. Casos associados à insuficiência renal crônica há principalmente perda de peso. Outros sinais que também são atribuídos a urolitíase são: cólica, relutância ao exercício, rigidez dos membros pélvicos e postura de urinar repetidamente [5].

A maioria dos autores descreve a bexiga urinária e a uretra, como os principais locais para a presença de urólitos [1], semelhante à localização do presente caso em que o urólito estava localizado na uretra causando obstrução total. No entanto outros autores contestam esta localização, pois cálculos renais e ureterais seriam mais comuns, mas raramente diagnosticados, pois geralmente são assintomáticos [1], além de que, uma proporção significativa dos cálculos que são encontrados na bexiga urinária pode ter origem na pelve renal, e foram levados pela urina através do ureter. Em um levantamento de 325 casos de urolitíase em equinos, $10 \%$ apresentaram cálculos em múltiplos locais [5]. Ruptura da bexiga urinária seguida de uroperitonite e nefrite como observado neste caso, são consequências comuns na urolitíase obstrutiva [5,6].

Os urólitos nos equinos são geralmente compostos por uma variedade de sais de cálcio hidratado e sais de carbonato de cálcio, magnésio e fósforo, sendo os compostos por estruvita menos comuns [1,5]. Uretrolitíase é pouco descrita em equinos e pode ser causada por cálculo de estruvita.

Declaration of interest. The authors declare no conflict of interest. The authors are responsible for the content and writing of the paper.

\section{REFERENCES}

1 Diaz-Espinera M., Escolar E., Bellanato J. \& Rodriguez M. 1995. Structure and composition of equine uroliths. Journal of Equine Veterinary Science. 15: 27-34.

2 Divers T.J. 2006. Urolitíase e doença obstrutiva. In: Smith P.B. (Ed). Medicina Interna de Grandes Animais. 3 ed. Barueri: Editora Malone, pp.841-843.

3 Duesterdieck-Zellmer K.F. 2007. Equine urolithiasis. Veterinary Clinics of North America: Equine Practice. 23: 613-629. 4 Ehnen S.J., Divers T.J., Gillette D. \& Reef V.B. 1990. Obstrutive nephrolithiasis and ureterolithiasis associated with chronic renal failure in horses: eight cases (1981-1987). Journal of the American Veterinary Medical Association. 197 : 249-253.

5 Laverty S., Pascoe J.R., Ling G.V., Lavoie J.P. \& Ruby A.L. 1992. Urolithiasis in 68 horses. Veterinary Surgery. 21: 56-62.

6 Saam D. 2001. Urethrolithiasis and nephrolithiasis in a horse. Canadian Veterinary Journal. 42: 880-883.

7 Trotter G.W., Bennett D.G. \& Behm R.J. 1981. Urethral calculi in five horses. Veterinary Surgery. 10: 159-162. 